\title{
Die Corona-Pandemie und der hysterische Diskurs nach Lacan
}

\author{
Eine Diskursbestimmung am Beispiel \#allesdichtmachen
}

\author{
Nils F. Töpfer (iD)
}

Angenommen: 26. November 2021 / Online publiziert: 21. Dezember 2021

(C) The Author(s), under exclusive licence to Springer Medizin Verlag GmbH, ein Teil von Springer Nature 2021

Zusammenfassung Im vorliegenden Beitrag werden die Ende April 2021 unter dem Hashtag \#allesdichtmachen veröffentlichten Video-Clips als ironisch und satirisch zugespitzte Charakterisierung des aktuellen Corona-Diskurses analysiert. Unter Bezug auf den hysterischen Diskurs nach Jacques Lacan ergibt sich folgender Ausgangspunkt für die Analyse: Bürger*innen als gespaltene Subjekte (\$) richten Appelle an den großen Anderen der Bundesregierung in der Erwartung, ihnen zu liefern (S1), was sie begehren (zum Beispiel absolute Sicherheit). Die von dem Anderen in der Folge entwickelten Corona-Maßnahmen (S2) können den fundamentalen Mangel (a) jedoch nicht aufheben und führen zu weiteren Appellen der Bürger*innen (\$). Die Verdrängung des Objekts klein a im hysterischen Diskurs wird in den Videos als Illusion vollständiger Sicherheit am Beispiel der Unvermeidbarkeit des Todes thematisiert. Das für den hysterischen Diskurs charakteristische Wandern des Begehrens resultiert in kontinuierlichen Appellen nach weiteren Maßnahmen. Die in den Videos vielfach selbst entwickelten Corona-Maßnahmen werden als Vervollständigung des Anderen und Identifikation mit dem Herrn gelesen. Angst wird mit dem hysterischen Begehren nach einem unerfüllten Begehren in Verbindung gebracht. Der Beitrag schließt mit Möglichkeiten der Diskursverschiebung vom hysterischen Diskurs zum Diskurs des Analytikers und von der paranoid-schizoiden zur depressiven Position nach Melanie Klein.

Dr. phil. Nils F. Töpfer $(\bowtie)$

Abteilung für Klinisch-Psychologische Intervention, Friedrich-Schiller-Universität Jena,

Humboldtstraße 11, 07743 Jena, Deutschland

E-Mail: nils.toepfer@uni-jena.de 


\title{
The COVID-19 pandemic and Lacan's discourse of the hysteric
}

A discourse characterization using the example of \#allesdichtmachen

\begin{abstract}
In this article the video clips uploaded under the hashtag \#allesdichtmachen at the end of April 2021 are analyzed as ironically and satirically exaggerated characterizations of the current coronavirus disease 2019 (COVID-19) discourse. Drawing on Lacan's discourse of the hysteric, the following starting point for the analysis is used: the citizens as divided subjects (\$) protest and demand that the other (the federal government) delivers (S1) what they desire (e.g. absolute security). The other subsequently produces solutions (S2, e.g. to issue measures against the COVID-19) which, however, do not solve the fundamental lack (a) but actually lead to further demands from the citizens $(\$)$. The suppression of object petit $a$ in the discourse of the hysteric is addressed in the video clips as the illusion of absolute security using the example of the inevitability of death. The drifting of desire, which is characteristic of the hysteric's social bond, results in demanding continuously new measures against the corona virus. The self-developed measures which are promoted in many video clips are interpreted as attempts at completion of the other and identification with the master. Anxiety is linked to the hysteric's desire for an unfulfilled desire. The article concludes with a discussion on how the discursive configuration can be challenged and evolves from the discourse of the hysteric to the discourse of the analyst and from the Kleinian paranoid-schizoid position to the depressive position.
\end{abstract}

\section{Die Aktion \#allesdichtmachen}

Ende April 2021 sorgte eine Internet-Aktion unter dem Hashtag \#allesdichtmachen für Furore. Fünfzig deutschsprachige Schauspieler und zwei Regisseure, darunter prominente Schauspieler wie Jan-Josef Liefers, Heike Makatsch, Ulrich Tukur, Nadja Uhl und viele mehr, kommentierten mit den Mitteln der Ironie und satirischen Zuspitzung in 53 Videos die Corona-Politik der deutschen Bundesregierung und die Medienberichterstattung zum Thema. Die Hashtags zählten am 22.04.2021 zu den meistverwendeten auf Twitter in Deutschland. Am 23.04.2021 berichtete die Tagesschau über die Internet-Aktion, die ,große Wellen geschlagen“ und einen „Proteststurm in den sozialen Medien“ ausgelöst habe.

In dem vorliegenden Beitrag werde ich den Diskurs zur Corona-Pandemie analysieren, der aus meiner Sicht in den Videos der Aktion \#allesdichtmachen thematisiert wird, indem ich auf den hysterischen Diskurs nach Lacan Bezug nehme. Beginnen möchte ich mit einem Auszug aus dem Kommentar auf der Website allesdichtmachen.de:

Vielmehr geht es uns um die Corona-Politik, ihre Kommunikation und den öffentlichen Diskurs, der gerade geführt wird. Wir üben Kritik mit den Mitteln von Satire und Ironie. Wenn man uns dafür auf massivste Art und Weise beschimpft und bedroht, ist das ein Zeichen, dass hier etwas ins Ungleichgewicht geraten ist .... Es geht um die Art, wie Staat und Bürger interagieren ... . (Wunder 2021) 
Daraus schließe ich, dass mit den Videos beabsichtigt wird, den Corona-Diskurs im Sinne einer ironisch und satirisch zugespitzten Diskursbestimmung zu charakterisieren, und dass die zum Teil heftigen Reaktionen in den sozialen Medien als Resultat und Ausdruck des spezifischen Diskurses betrachtet werden.

\section{Lacans Diskurstheorie und das gespaltene Subjekt}

Nach Lacan bezieht sich Diskurs auf das soziale Gefüge, darauf, wie das Subjekt darin situiert ist, und wie diese Position es dem Subjekt ermöglicht, die eigene Gespaltenheit zu bewältigen bzw. eine Konfrontation damit zu verhindern (Lacan 1993). Lacan unterschied vier Diskurse: den Diskurs des Herrn, den Diskurs der Universität, den hysterischen Diskurs und den Diskurs des Analytikers (Lacan 2007). Die vier Diskurse haben alle die gleiche Struktur und bestehen aus vier Positionen: der Agent, der Andere, die Wahrheit, die Produktion (Abb. 1).

Alle Positionen auf der linken Seite sind im jeweiligen Diskurs aktiv, das heißt, sie senden eine Botschaft, während die Empfänger der Botschaft die Positionen auf der rechten Seite einnehmen. Die oberen Positionen stehen für das Manifeste in einem Diskurs, während die unteren Positionen, getrennt durch die gestrichelte Linie, das Latente, das Verborgene oder Verdrängte darstellen. Die obere linke Position des Agenten ist die aktivste und mächtigste. Der Agent richtet sich an einen Anderen, was durch den horizontalen oberen Pfeil angezeigt wird. Die gesendete Botschaft wird jedoch nie so empfangen wie beabsichtigt. Hier kommt Lacans Auffassung zum Ausdruck, dass die Sprache und das Sprechen nicht beherrscht werden können und jede Kommunikation ein Missverständnis ist bzw. als solches endet (Klepec 2016). Der Pfeil von oben nach unten rechts in der Abbildung zeigt an, dass die Ansprache des Agenten an den Anderen ein Produkt zur Folge hat, das den Agenten antreibt (diagonaler Pfeil von unten rechts nach oben links). In jedem Diskurs werden die Handlungen des Agenten durch eine verborgene Wahrheit motiviert, was durch den Pfeil von unten nach oben auf der linken Seite verdeutlicht wird. Diese Wahrheit wirkt sich auch auf die Position des Anderen aus (diagonaler Pfeil von unten links nach oben rechts), während die Produktion eine disjunktive Position in Bezug auf die Wahrheit einnimmt, die den Diskurs in Gang setzt (kein Pfeil von Produktion zu Wahrheit; Vanheule 2016).

Die vier Positionen werden in den vier Diskursen immer von vier Elementen eingenommen: $S_{1}-$ Herrensignifikant, $S_{2}-$ Wissen, a - „object petit a“, $\$$ - das gespaltene Subjekt. Diese vier Elemente bleiben immer in dieser Reihenfolge und nehmen lediglich unterschiedliche Positionen in den vier Diskursen ein, sodass es

Abb. 1 Diskurspositionen nach Lacan (2007)

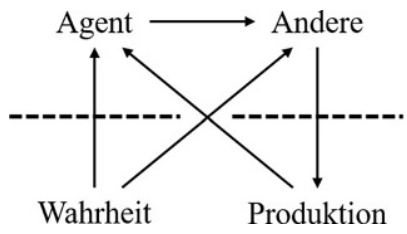


nicht 24 mögliche Kombinationen, sondern vier Diskurse gibt, die sich durch Drehen der Elemente im Uhrzeigersinn oder gegen der Uhrzeigersinn ergeben (Klepec 2016). Ich werde mich im Folgenden auf den hysterischen Diskurs fokussieren, der mir - und anderen (Johanssen 2020) - für den Corona-Diskurs besonders relevant erscheint.

\section{Der hysterische Diskurs und die Corona-Pandemie}

Die vier Positionen stehen im hysterischen Diskurs in folgendem Zusammenhang: Die Position des Agenten besteht aus dem gespaltenen Subjekt (\$), das Appelle an den Anderen richtet, von dem angenommen wird, dass er eine Antwort (S1) auf das hat, was das Subjekt umtreibt und irritiert. Dieser Diskurs verdrängt die Wahrheit, dass alles Begehren auf einem Mangel beruht, der nicht behoben werden kann (a), und führt typischerweise zur Produktion von Narrativen (S2), die den fundamentalen Mangel ( $a$ ) nicht auflösen können und folglich weitere Appelle des gespaltenen Subjekts hervorrufen (\$; Vanheule 2016) (siehe Abb. 2).

Auf den Corona-Diskurs ließe sich das wie folgt übertragen: Die Bürger*innen fordern fortwährend, dass die Bundesregierung das liefert, was die gespaltenen Subjekte begehren (zum Beispiel absolute Sicherheit). Als Reaktion auf die Appelle erlässt die Bundesregierung immer weitere, neue Corona-Maßnahmen. Die im hysterischen Diskurs unterdrückte Wahrheit ist nach Lacan das Objekt klein a (Object petit a), der Grund des Begehrens. Es ist die Leerstelle, die ein (verlorenes) Objekt einnehmen kann, das heißt die Position, die es ermöglicht, dass das Objekt nicht erreichbar und damit begehrt ist (Jacobsen 2020). Bewusst wurde aus folgendem Grund das Wort „,verlorenes“ in Klammern gesetzt: „Das Objekt a ist kein Objekt, das wir verloren haben, da dies bedeuten würde, dass wir es finden und unser Begehren befriedigen könnten. Es ist vielmehr das ständige Gefühl, dass in unserem Leben etwas fehlt. Wir suchen ständig nach Erfüllung, nach Wissen, nach Besitz, nach Liebe, und wann immer wir diese Ziele erreichen, wollen wir immer noch etwas mehr; wir können es nicht genau lokalisieren, aber wir wissen, dass es da ist“" (Homer 2016, S. 100; Übersetzung des Verfassers).

Für das gespaltene Subjekt hält ein (verlorenes) Objekt, das diese Position einnimmt, das Versprechen, durch das Objekt seine Lücke aufzufüllen und dadurch vollständig zu werden. Wenn das Objekt klein a wie im hysterischen Diskurs verdrängt wird, vermeidet das Subjekt, sich seinem Begehren und letztlich dem ontologischen Mangel im Zentrum seines Seins zu stellen: Der Mangel ist letztlich nicht aufzuheben! (Jacobsen 2020). Bereits an dieser Stelle wird deutlich, dass die Formulierung

Abb. 2 Hysterischer Diskurs. Erklärung siehe Text

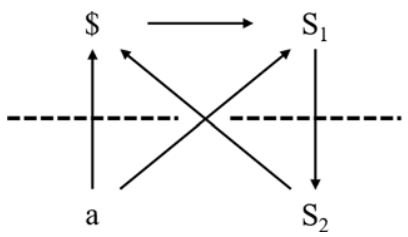


„allesdichtmachen“ mit Lacan, dem „Topologen der Löcher“, auf die Verleugnung von etwas basal Menschlichem verweist: Begehren ist immer das Begehren nach etwas, das fehlt (Homer 2016). ${ }^{1}$

\title{
Die Illusion vollständiger Sicherheit am Beispiel der Unvermeidbarkeit des Todes
}

Der hysterische Diskurs im Kontext der Corona-Pandemie ist insofern illusionär, als der Andere nicht alle nötigen Informationen liefern und keine vollständige Sicherheit gewährleisten kann. Die Illusion vollständiger Sicherheit wird in den \#allesdichtmachen-Videos im Zusammenhang mit der Unvermeidbarkeit des Todes thematisiert. So zeigt sich Roland Düringer (2021) in seinem Video froh darüber, ,dass der Staat meine Gesundheit zu einer öffentlichen Angelegenheit gemacht hat", obwohl er früher dachte, selbst Verantwortung für seine Gesundheit übernehmen zu können.

\begin{abstract}
Aber heute, gut informiert, weiß ich es besser. Mein Schicksal, das liegt in Ihrer Hand. Krankheit muss nicht sein. Und selbst der Tod ist ja lediglich ein Fehler der Schöpfung. Wenn wir uns brav anstrengen, dann können wir gemeinsam mit der modernen Wissenschaft diesen Fehler korrigieren. Wer in Zukunft schon mit 85 Jahren in die Kiste hüpft, der hat eigentlich nur ein SoftwareUpdate vergessen. Also, bleiben Sie mir bitte gesund, befolgen Sie brav die Maßnahmen der Bundesregierung und bescheren Sie so mir und auch Ihnen ein ewiges Leben!
\end{abstract}

Ein Großteil der Video-Clips beginnt mit der Offenbarung, verunsichert oder besorgt zu sein oder Angst zu haben. Die Corona-Maßnahmen werden damit in einen Zusammenhang mit Versuchen der Angstbewältigung gestellt, für die man nicht selbst, sondern der Andere verantwortlich ist. Indem indirekt auf die ultimative Angst vor dem Tod Bezug genommen wird und die Maßnahmen als Versprechen eines ,ewigen Lebens“ idealisiert werden, müssen alle Maßnahmen zum Scheitern verurteilt sein. Die unrealistischen Hoffnungen, die in die Maßnahmen gesetzt werden, werden damit satirisch kritisiert, im Sinne von „Der Andere kann keine Antwort auf den Tod bzw. die Angst vor dem Tod haben!“"

Auch Ulrich Tukur (2021) thematisiert in seinem Video den Tod. Er rezitiert das „Schlußstück“ von Rainer Maria Rilke und fügt daran an:

Angesichts des großen Todes, der uns alle umsteht und den nicht einmal die fürsorgliche Politik engagierter deutscher Regierungsbeamter wegzuadminstrieren im Stande sein wird, scheint es mir vollkommen irrelevant, ob eine Tankstelle als systemrelevant offen bleibt oder ein Wirtshaus als systemirrelevant geschlossen wird.

Er fordert, ,ausnahmslos jede menschliche Wirkungsstätte und jeden Handelsplatz“ zu schließen, ,,vor allem die Supermärkte“, denn ,sind wir erst am Leibe und nicht nur an der Seele verhungert und allesamt mausetot, entziehen wir auch dem Virus ... die Lebensgrundlage“. Tukur thematisiert ebenfalls - allerdings explizit und

\footnotetext{
1 Ich danke Prof. Dr. Timo Storck für diesen passenden Einfall.
} 
nicht im Sinne der omnipotenten Verleugnung wie im Video von Düringer - die Unmöglichkeit, den Tod „,wegzuadministrieren“. Indem er argumentiert, dass nur dann keine Gefahr mehr von dem Virus ausgeht, wenn auch keine Menschen mehr leben, weist er auf die Unmöglichkeit hin, eine Ansteckungsgefahr völlig auszuschließen.

\section{Die Erschütterung der symbolischen Ordnungen der Gerechtigkeit und Gleichheit}

Durch die extremen Positionen „,Jeder könnte ewig leben!“ (Düringer) und „Alle müssten erst sterben“ (Tukur) thematisieren die Videos nicht nur die Unmöglichkeit absoluter Sicherheit und die Frage nach einem annehmbaren (Un-)Sicherheitsmanagement; die Videos thematisieren auch Fragen der (Un-)Gerechtigkeit und (Un-)Gleichheit. Auch in anderen Videos wird dieses Thema adressiert, zum Beispiel von Thorsten Merten (2021, „Aber ich mache mir Sorgen, weil viele egoistisch sind und keinen Abstand halten. Warum können diese Leute nicht einfach auch in große Wohnungen, Häuser oder Villen ziehen, um Abstand zu halten?!“) und von Nadine Dubois (2021):

So viele Menschen da draußen krempeln die Ärmel hoch und packen mit an, damit ich hier sitzen und mich und andere schützen kann. Das finde ich gut. Nicht gut finde ich, wenn diese Menschen dann am Wochenende rausgehen aus ihren kleinen dunklen Wohnungen und sich in den Park setzen. Dann gehe ich auf meinen Balkon, mache davon Fotos und schreibe auf Twitter, dass sie schrecklich egoistisch sind. Und dann gehe ich auf meine Dachterrasse und lasse mir die Sonne ins Gesicht scheinen und fühle mich gut, weil ich mich und andere schütze.

Wie bereits Küchenhoff (2020) dargestellt hat, legt die Corona-Krise die bereits vorbestehende gesellschaftliche Ungerechtigkeit bloß und stellt die symbolischen Ordnungen der Rechts- und Chancengleichheit infrage.

\section{Das Wandern des Begehrens und die kontinuierliche Produktion weiterer Corona-Maßnahmen}

Ich möchte nun darauf zurückkommen, dass das Objekt klein a im hysterischen Diskurs verdrängt wird. Die Funktion des Objekts klein a wird jedoch sichtbar, durch die Wanderung des Begehrens von einem (verlorenen) Objekt zum nächsten, jeweils verbunden mit der illusorischen Erwartung, dass jedes neue begehrte Objekt das symbolisieren kann, was dem Subjekt fehlt (Jacobsen 2020). Viele Videos scheinen mir satirisch auf dieses Wandern des Begehrens in Form der Notwendigkeit von immer neuen Corona-Maßnahmen zu verweisen.

Deshalb unterstütze ich die Corona-Maßnahmen, egal, welche da mal wieder an der Reihe sind. Damit wir alle sicher sind. Aber, um wirklich sicherzugehen, muss man jeden Tag nachschauen, denn was heute sicher ist, ist morgen vielleicht schon unsicher. (Duken 2021) 
Der größte Teil der Videos thematisiert das Wandern des Begehrens jedoch insofern, als selbstständig eigene Maßnahmen entwickelt und beworben werden, zum Beispiel die 2-Tüten-Atmung (Richy Müller 2021; Einatmen aus einer Tüte, Ausatmen in eine andere Tüte) oder die Inzidenzregelung in der Familie (Kathrin Osterode 2021; ,Jeden Morgen schreiben wir hier den gültigen Inzidenzwert auf diese Tafel, um das Ganze für die Kinder spürbar und erlebbar zu machen. Und deshalb hat der Wert auch ganz konkrete Auswirkungen auf das, was wir als Familie dürfen und was nicht"). Einerseits ließen sich diese selbstentwickelten Maßnahmen als Kritik der Videos an der subjektiv erlebten Absurdität einiger Maßnahmen verstehen oder - im Falle des Videos von Kathrin Osterode - als polemische Kritik an den negativen Konsequenzen, welche die Maßnahmen für einige bedeuten können (,Ab einem Wert von 250 wird das erste Kind zur Adoption freigegeben; ab einem Wert von 300 dann das zweite."). Mit Bezug auf den hysterischen Diskurs könnten die selbstentwickelten Maßnahmen andererseits auch als Ausdruck davon aufgefasst werden, dass die bisherigen Maßnahmen nicht ausreichen und es daher immer weitere und neue Maßnahmen braucht.

\section{Die Vervollständigung des Anderen und die Identifikation mit dem Herrn}

Aber warum werden die Maßnahmen selbst entwickelt, anstatt neue Maßnahmen zu fordern? Hier scheint mir ein Rückgriff auf Lacans Überlegungen zur Hysterie aufschlussreich. Nach Lacan konstituiert sich das hysterische Subjekt als das Objekt, das der Andere begehrt, da ihm/ihr dadurch ein Platz im Anderen sicher ist (Fink 1999). Durch die Identifikation mit dem Anderen begehren Hysteriker*innen, als wären sie der Andere. Die bekannte Formulierung Lacans, dass das Begehren das Begehren des Anderen ist, kann auch so verstanden werden, dass das Begehren des Anderen als eigenes Begehren übernommen wird. Die selbstentwickelten Corona-Maßnahmen könnten daher mit Lacan als Versuch gelesen werden, das begehrte Objekt des Anderen (zum Beispiel der Bundesregierung) zu verkörpern und den Anderen damit zu ,,vervollständigen“ und zu „komplettieren“. Es erscheint mir plausibel, dass in den Videos der Internet-Aktion \#allesdichtmachen mithilfe der satirisch überzogenen selbsterfundenen Maßnahmen eine (Über-)Identifizierung mit den Corona-Maßnahmen und eine daraus resultierende „Objektifizierung“ der Bürger*innen kritisiert werden sollen.

\section{Exkurs zur Psychodynamik zwischen hysterischem Subjekt und dem Anderen}

An dieser Stelle könnte sich die Frage stellen, ob die Aktion \#allesdichtmachen nun vorwiegend die hysterischen Bürger*innen, zum Beispiel ihre (Über-)Identifizierung mit den Corona-Maßnahmen, kritisiert oder eher die Bundesregierung, die hysterische „Objekte“ produziert, die den Appell nach Maßnahmen zur (vermeintlichen) Angstreduktion erheben, dem dann entsprochen werden soll. Mir scheinen die Video-Clips keine eindeutige Antwort zu geben. Passenderweise wird diese Frage in dem eingangs zitierten Auszug aus der Stellungnahme auf der Website weitgehend offengelassen und die Aufmerksamkeit stattdessen auf ,die Art, wie Staat und Bürger interagieren“, gerichtet. Viele Video-Clips sind aus der Position der hyste- 
rischen Bürger*innen vorgetragen und kritisieren gleichzeitig, von dem Anderen in dieser Position festgehalten zu werden: Die hysterischen Bürger*innen wollen, dass man ihnen sagt, was sie machen sollen, um sich sicher zu fühlen, und der Andere folgt diesem Appell. Auch Jörg Bundschuh (2021) positioniert die Bürger*innen in seinem Video-Clip in dieser Weise:

Da draußen sind jetzt so viele Menschen, die nicht wissen, was sie tun sollen.

Diese Menschen brauchen Aufklärung, Sie brauchen Werbespots für's Hände-

Waschen und für's Maske-Tragen. Sie brauchen Plakate, auf denen steht, dass sie diszipliniert sein sollen. Diese Menschen wollen diese Plakate. Das ist jetzt so wichtig.

Indem er anmerkt „Die Menschen sind so dumm, man muss ihnen genau sagen, was sie machen sollen“, weist er daraufhin, dass die Bundesregierung durch Werbespots und Plakate die Bürger*innen dann auch als genauso dumm verkauft. Es wirkt wie das „Henne-Ei-Problem“: Was war nun zuerst da? Anstatt diese Frage aufzulösen, werde ich im Folgenden stattdessen die Dynamik zwischen dem hysterischen Subjekt und dem Anderen genauer beleuchten, um herauszuarbeiten, wer daraus was für sich zieht (und was auch nicht), und um anhand der Fallstricke des hysterischen Diskurses die Möglichkeiten der Diskursverschiebung vorzubereiten.

Lacan zufolge nimmt das gespaltene Subjekt im hysterischen Diskurs die dominante Position ein und wendet sich an den Anderen. In der Psychotherapie fragen Hysteriker*innen, was sie haben und was mit ihnen los ist, und erwarten Antworten auf diese Fragen von ihren Therapeut*innen. Wenn sich Therapeut*innen den Forderungen beugen und ihnen Wissen liefern, wird dieses Wissen nur kurzzeitig Erleichterung verschaffen, und schon kurz darauf werden die Hysteriker*innen die Lücke und den Mangel im Wissen der Therapeut*innen suchen oder ein neues Symptom präsentieren: „Sie [die Hysterikerin] wird zur Herrin über das Wissen des Analytikers und bringt ihn dazu, dieses Wissen so schnell wie möglich zu produzieren“ (Fink 1999, S. 132; Übersetzung des Verfassers). Als ein Beispiel ließe sich Freuds Fallgeschichte Dora anführen. Freud positionierte sich als der große Andere, der unermüdlich nach einem bestimmten Objekt suchte, das Doras unbewusstes Begehren erfüllt, und durch seine Interventionen und Deutungen Dora von seinem Wissen zu überzeugen versuchte (Van Haute und Geyskens 2016). Wenig überraschend blieben diese Versuche erfolglos, und Dora brach die Therapie nach wenigen Monaten ab.

Das Fordern, Befehlen und Kommandieren der Hysteriker*innen ist jedoch gleichzeitig ein Ausdruck ihrer Abhängigkeit vom Anderen, um zum Subjekt zu werden. Die Lücke bzw. den Mangel im Wissen des Anderen zu finden, ermöglicht dem hysterischen Subjekt ,die Rolle der Ausnahme, des lebenden Beweises, dass sie das Wissen des Analytikers ergänzen oder vervollständigen kann“ (Fink 1999, S. 132). Die phantasmatische Position, den Anderen vervollständigen zu können, impliziert auch die Weigerung, den eigenen Mangel bzw. die eigene Kastriertheit anzuerkennen (Dor 1999). Hier wird deutlich, was die Protagonisten von einer solchen Dynamik haben: Beide, das hysterische Subjekt und die Person in der Position des Anderen, haben (angeblich) das, was dem jeweils anderen fehlt. Zwei Ansatzpunkte für Diskursverschiebungen lassen sich damit bereits zusammenfassen. Einerseits 
muss die Frage „Was ist los mit mir?“ an das hysterische Subjekt zurückgegeben werden, im Sinne von „Was willst $d u$ ?“ (Fink 1999). Andererseits würde sich der Diskurs verschieben können, wenn das hysterische Subjekt anerkennen würde, nicht der Phallus zu sein, bzw. der Andere anerkennen würde, nicht den Phallus zu haben (Dor 1999).

Sexualität eignet sich als ein eindrückliches Beispiel, um weiter zu verdeutlichen, wie der Andere in die Dynamik des hysterischen Diskurses eingespannt wird und wie er/sie zur Aufrechterhaltung des Diskurses beiträgt. Sexualität ist ein häufiger Gegenstand hysterischer Klagen und Beschwerden. Mit solchen sexuellen Klagen können hysterische Frauen bei Männern gut landen, da sie deren männliche Potenz wortwörtlich infrage stellen. So entfaltet sich ein tragisches Missverständnis: Die charakteristischen hysterischen Appelle (zum Beispiel „Er kann mich nicht zum Orgasmus bringen“) führen dazu, dass sich der Mann in seiner Männlichkeit angezweifelt fühlt und durch übermäßige Bemühungen versucht zu beweisen, dass er nicht impotent ist. Diese Bemühungen können jedoch den Anstoß für weitere hysterische Klagen bieten (,Er will immer mit mir Sex haben, ohne zu fragen, ob ich überhaupt Lust habe“; Dor 1999, S. 91). Wenngleich diese Dynamik nicht explizit in \#allesdichtmachen thematisiert wird, so ließe sie sich ohne Weiteres auf den Corona-Diskurs beziehen. ${ }^{2}$

\section{,... ich merke, wie meine Angst nachlässt, und das macht mir Angst“": Angst im hysterischen Diskurs}

Wie bereits angemerkt, ist das Objekt klein a die Leerstelle, die ein (verlorenes) Objekt einnehmen kann, welches das Versprechen hält, das gespaltene Subjekt zu vervollständigen. Angst ist nach Lacan in diesem Zusammenhang ein Ausdruck davon, kein (verlorenes) Objekt zu haben: „Es ist der Mangel eines symbolisierbaren Mangels: Angst ist der Moment, in dem das Subjekt dem objet petit a zu nahe kommt. In der hysterischen sozialen Bindung entsteht also Angst bei der Aussicht, dass der „Andere“ das liefert, was das Subjekt begehrt, und treibt das Begehren voran“ (Jacobsen 2020, S. 5; Übersetzung des Verfassers). Diese Art der Angst scheint mir das Video von Volker Bruch (2021) zu thematisieren:

Mein Name ist Volker Bruch. Ich bin Schauspier und ich habe Angst, aber ich merke, wie meine Angst nachlässt, und das macht mir Angst. Ein Jahr lang hatte ich durchgehend Angst, doch diese Angst lässt jetzt nach. Und das macht mir Angst. Ich will wieder mehr Angst haben. Denn ohne Angst habe ich Angst. Deshalb appelliere ich an unsere Regierung: Macht uns mehr Angst. Die Menschen im Land brauchen diese Angst jetzt. Liebe Regierung. Lasst uns in dieser

\footnotetext{
${ }^{2}$ Hier geht es weder um eine Generalisierung von Geschlechterrollen noch um die Hysterie als nosologische ,pathologische“ Kategorie, sondern um eine Struktur, durch die Begehren einen Platz in der Ökonomie des Diskurses erhält. Der hysterische Diskurs weist insofern die elementarste Form des Sprechens auf, als das sprechende Subjekt als solches hysterisch ist $(\$ \rightarrow$ S1). Daher stellt die „Hysterisierung“ der Analysand*innen, unabhängig von deren Persönlichkeitsstruktur, in der Psychoanalyse auch ein notwendiges Übergangs- oder Zwischenstadium zum Diskurs des Analytikers dar (siehe Abschnitt ,,Vom hysterischen Diskurs zum Diskurs des Analytikers").
} 
Lage nicht allein. Es ist jetzt so wichtig, dass wir alle genug Angst haben. Blei-

ben Sie gesund, und halten Sie sich an Ihrer Angst fest.

Die scheinbar paradoxe Aussage „Ich merke, wie meine Angst nachlässt, und das macht mir Angst“" wirkt auf mich verständlich, wenn die erste Angst auf die Sorge und Irritation bezogen wird, die zu Appellen nach Corona-Maßnahmen führt, die an den Anderen der Bundesregierung gerichtet werden, und die zweite Angst als Angst im eben beschriebenen Lacan'schen Sinne, die entsteht, wenn der Andere das eigene Begehren wirklich erfüllen könnte. Lacan spricht von dem hysterischen Begehren nach einem unerfüllten Begehren. Das resultierende Wandern des Begehrens im hysterischen Diskurs ist insofern produktiv, als es den Anderen dazu anhält, beständig die Antworten auf die Appelle des Subjekts zu verbessern, zugleich konservativ, als die Unterdrückung von Objekt klein a verhindert, dass die diskursive Konfiguration hinterfragt wird (Jacobsen 2020). Auf eine Kritik der diskursiven Konfiguration scheinen mir die Videos von \#allesdichtmachen abzuzielen, indem sie das Hysterische des Diskurses überspitzt hervorheben.

\section{Möglichkeiten der Diskursverschiebung}

\section{Zur fehlenden Subversion der Corona-Proteste: Kritik als Herrensignifikant}

Im Zusammenhang mit den Möglichkeiten der Diskursverschiebung könnte sich die Frage stellen, wie aus der Perspektive der Lacan'schen Diskurstheorie das subversive Potenzial der Corona-Proteste, zum Beispiel durch die Querdenker-Bewegung, einzuschätzen ist. Die Antwort: Gleich null. Das einzige, wozu traditionelles revolutionäres Begehren nach Lacan führen kann, ist der Diskurs des Herren, von dem der Diskurs der Universität insofern eine pervertierte Form darstellt, als er dazu dient, Herrensignifikanten zu propagieren, auf denen der Diskurs basiert (Bracher 1993). Der Herrensignifikant in den Corona-Protesten ist die Kritik selbst: „Kritik bekommt dadurch einen ethischen Eigenwert, sie wird sich selbst zum Hauptzweck" (Nachtwey et al. 2020, S. 60). Im Diskurs der Universität ist die Wissensproduktion letztlich auf eine Bestätigung ,,vorgefertigten Wissens“ ausgerichtet. So zeigt die Forschung zu den Corona-Protesten zum Beispiel, dass die Glaubwürdigkeit der alternativen Informationsquellen, auf die sich die Kritik stützt, einfach daraus abgeleitet wird, dass Kritik geäußert wird: „Die Glaubwürdigkeit der Kritik ist also eigentlich eine Glaubwürdigkeit durch Kritik“ (Nachtwey et al. 2020, S. 60). Der Diskurs der Universität steht insofern sozialem Wandel im Wege als die Befriedigung, die aus dem ,produzierten“ Wissen gezogen wird, dazu verleitet, einfach in diesem Wissen zu schwelgen. Im Falle der Corona-Proteste resultiert diese Befriedigung aus der „Selbstdarstellung als Kritiker:in“ (Nachtwey et al. 2020, S. 60f). Wenn klassische revolutionäre Rhetorik und kritische Bewegungen Risiko laufen, den Diskurs des Herren zu wiederholen, muss nach Lacan ein Diskurs mit entgegengesetzter Struktur gefördert werden: der Diskurs des Analytikers. 


\section{Vom hysterischen Diskurs zum Diskurs des Analytikers}

Vor dem Hintergrund, dass das Wandern des Begehrens ein Hinterfragen der diskursiven Konfiguration verhindert, wäre „das Schlimmste“, das im hysterischen Diskurs passieren könnte, dass der Andere der Kritik nachgibt und die Position des Herrn aufgibt, fortwährend neue Lösungen hervorzubringen (Jacobsen 2020). Dadurch würde das Subjekt dazu angehalten, Verantwortung für seine eigenen Entscheidungen zu übernehmen und sich letztendlich seinem eigenen Begehren zu stellen.

Dass ein Eingeständnis der eigenen „Kastriertheit“ durch den Herrn den hysterischen Diskurs erschüttern würde, wird unter anderem im Video von Ken Duken (2021) thematisiert:

Denn wenn sich diejenigen, die sich eigentlich sicher sein sollten, dass wir sicher sind, unsicher sind, ob wir nicht vielleicht doch unsicher sind, wie soll sich dann jemand wie ich sicher fühlen?! Und sicherlich ist Sicherheit am allerwichtigsten.

Diejenigen in der Position des Herrn wie Politiker*innen und Expert*innen sollten dem Anspruch auf ,Allwissenheit“ abschwören. Eingeständnisse der Begrenztheit des eigenen Wissens ähneln nach Johanssen (2020) dem Diskurs des Analytikers. Lacan zufolge nehmen Analytiker*innen zwar die Position des Anderen an, aber ihr Begehren bleibt rätselhaft. Dadurch wird ein Prozess angestoßen, in dem Analysand*innen versuchen herauszufinden, was ihre Analytikerin von ihnen will. Indem sich die Analytikerin den Grenzen ihres eigenen Wissens verpflichtet fühlt, ermöglicht sie es ihren Analysand*innen, ihre eigenen Wünsche, Konflikte und Fantasien durchzuarbeiten. Vanheule (2016) fasst dies unter Einbezug der vier Diskurspositionen wie folgt zusammen: „Indem der Analytiker den Platz des Objekt klein a einnimmt (a), schafft er einen Raum, in dem via freier Assoziation die Subjektgespaltenheit artikuliert werden kann (\$). Um der Singularität des Patienten große Aufmerksamkeit zu schenken, legt der Analytiker vorgefasste Vorstellungen über Patienten und Pathologien (S2) beiseite, so dass Herrensignifikanten, die die Subjektivität des Patienten kennzeichnen (S1), formuliert werden können, was die Positionierung des Analytikers qua Objekt klein a (a) fördert" (S. 3; Übersetzung des Verfassers; Abb. 3).

Während Analytiker*innen nach Lacan den Diskurs des Analytikers einnehmen sollten, werden Analysand*innen unabhängig von ihrer Persönlichkeitsstruktur in den hysterischen Diskurs hineingezogen bzw. müssen ,hysterisiert“ werden (Fink 1995; siehe auch das sprechende Subjekt als solches ist hysterisch, Abschnitt „Exkurs zur Psychodynamik zwischen hysterischem Subjekt und dem Anderen“"). So

Abb. 3 Diskurs des Analytikers. Erklärung siehe Text

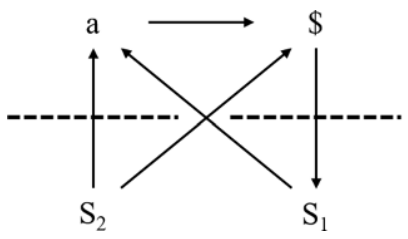


gesehen ist der hysterische Diskurs ein Übergangs- oder Zwischenstadium zum Diskurs des Analytikers. Dabei gilt es, aus therapeutischen Gesichtspunkten jedoch Folgendes zu berücksichtigen (Fink 1999): Einerseits könnten wir es als Analytiker*innen begrüßen, dass Analysand*innen im hysterischen Diskurs sehr aufmerksam für unser Begehren sind, es Ihnen leicht fällt, Hilfe anzunehmen und sich ihre Abhängigkeit von uns einzugestehen. Andererseits wollen sie sich eigentlich einer analytischen Arbeit verweigern: Sie wollen die Antworten von uns, und sie werden die Analyse erfolgreich boykottieren, wenn wir uns darauf einlassen sollten, sie mit Wissen zu füttern. Es hängt demnach alles davon ab, die Begrenztheit des eigenen Wissens anzuerkennen, die hysterisierten Analysand*innen arbeiten zu lassen und die Subjektgespaltenheit genau an solchen Stellen zu markieren, an denen die Spaltung zwischen Bewusstem und Unbewussten deutlich wird, zum Beispiel durch Freud'sche Versprecher und Fehlhandlungen, Träume usw. Entsprechend argumentiert Johanssen (2020), dass Bürger*innen und Experten*innen mit Bezug auf die Corona-Pandemie die Position des Analytikers einnehmen sollten, indem sie die Begrenztheit allen Wissens anerkennen und aufarbeiten, inwiefern ihre paranoidschizoiden und depressiven Formen des In-Beziehung-Tretens ihre Reaktionen auf die COVID-19-Pandemie beeinflussen.

\section{Von der paranoid-schizoiden zur depressiven Position im Corona-Diskurs}

Ergänzend zur Verschiebung hysterischer Diskurs $\rightarrow$ Diskurs des Analytikers ließen sich Interventionen zu einem veränderten Corona-Diskurs auch im Sinne paranoidschizoide Position $\rightarrow$ depressive Position nach Melanie Klein (2019) konzeptualisieren. Der für die paranoid-schizoide Position typische Spaltungsmodus als primitiver Abwehrmechanismus scheint mir in mehreren Videos von \#allesdichtmachen thematisiert zu werden, zum Beispiel von Martin Brambach (2021):

Ich bin Martin Brambach, Schauspieler, und ich habe im letzten Jahr angefangen, solidarisch mit dem Finger auf andere Leute zu zeigen. Ich bin ein eher unsicherer Mensch und brauche klare Regeln. Und es tut mir gut, wenn ich andere darauf hinweisen kann, was sie falsch machen. Und es gibt ja ständig neue Maßnahmen und Regelungen. Ich halte mich immer auf dem Laufenden. Dann bin ich der Erste, der das weiß, und kann den Menschen in meiner Umgebung sagen, was sie falsch machen. Besonders freue ich mich, wenn ich jemanden ohne Maske erwische. Den mache ich fertig. Dem sage ich dann, dass er ein rücksichtsloses Arschloch ist. DAS ist für mich gelebte Solidarität. Weil am Ende ist das gut für uns alle. Bleibt gesund. Bleibt solidarisch. Haltet euch an die Corona-Maßnahmen der Bundesregierung.

Nach Melanie Klein (2019) soll durch Spaltung das Gute vom Bösen getrennt gehalten werden, um das Gute vor dem Bösen zu schützen. Durch Projektion des Bösen kann dieses im Außen verortet und dort ,bekämpft“"werden: Dadurch kann das Mitdem-Finger-auf-andere-Zeigen und Beleidigen (,,rücksichtsloses Arschloch“) als solidarisch rationalisiert werden. Auch im Video von Nadine Dubois (2021) findet sich diese Spaltung wieder (,Dann gehe ich auf meinen Balkon, mache davon Fotos und schreibe auf Twitter, dass sie schrecklich egoistisch sind."). Schuldzuweisungen sind 
insgesamt ein wiederkehrendes Motiv in den Videos (Richy Müller 2021; „Denn es gibt so viele Egoisten und Undisziplinierte, und die sind doch daran schuld, dass alles so schlimm ist.“). Die paranoid-schizoide Position stellt eine Abwehrformation gegen existenzielle Bedrohungen dar, wie sie durch die Corona-Pandemie mobilisiert werden. In diesem Sinne leben wir nach Johanssen (2020) in paranoid-schizoiden Zeiten, die vom Thema des Todes überschattet werden und die Bevölkerung spalten: die Gesunden und die Kranken, die Jungen und die Alten, die Genesenen und diejenigen, die noch gefährdet sind, die Toten und die Lebenden und so weiter.

Zentral für die paranoid-schizoide Position sind paranoide Ängste und Fantasien. Paranoia stellt eine Abwehr angesichts schwer fass- und greifbarer Ängste dar: Aufgrund seiner Unsichtbarkeit eignet sich das Corona-Virus dazu, durch Verschwörungstheorien erklärt zu werden (Johanssen 2020). Für ein Virus, das so schwer kontrollierbar ist, Erklärungen und Verantwortliche zu finden sowie vermeintliche Kausalitäten herzustellen, kann ein Gefühl von Erleichterung gerade bei solchen Menschen bewirken, denen es besonders schwerfällt, die aktuell fundamentale Unsicherheit und Ungewissheit auszuhalten (Johanssen 2020).

Eine Annäherung an die depressive Position würde erfordern, sowohl die schreckliche Realität der aktuellen Krise zu sehen als auch positive Gefühle zulassen zu können, wie zum Beispiel die Entschleunigung des Alltags. In der depressiven Position kann das Nebeneinander ausgehalten und auf einseitige Narrative verzichtet werden. Die \#allesdichtmachen-Videos scheinen besonders die Einseitigkeit anzuprangern, wie schon aus dem Text auf der Website der Aktion deutlich wird: „Wenn man uns dafür auf massivste Art und Weise beschimpft und bedroht, ist das ein Zeichen, dass hier etwas ins Ungleichgewicht geraten ist." Die Reaktion auf die Aktion scheinen die Macher*innen somit als einen Ausdruck des Diskurses zu betrachten, den sie in ihren Videos überspitzt darstellen. Sie können einerseits als defensive Reaktion auf die Infragestellung des Anderen in der Position des Herrn und andererseits als Ausdruck eines paranoid-schizoiden Diskurses aufgefasst werden - neben einer Vielzahl weiterer, sicherlich auch vielfach berechtigter Gründe für Kritik und Empörung.

Interessenkonflikt N.F. Töpfer gibt an, dass kein Interessenkonflikt besteht.

\section{Literatur}

Bracher M (1993) Lacan, discourse, and social change. Cornell University Press, New York

Brambach M (2021) \#allesdichtmachen. https://www.youtube.com/watch?v=BogwFLDp7K8\&list= PLmb04gmYSul51BPqW1J93RgI1LYTCV6DF\&index=10. Zugegriffen: 15.12.2021

Bruch V (2021) \#allesdichtmachen. https://www.youtube.com/watch?v=sOCi3B9wJ5U. Zugegriffen: 15.12.2021

Bundschuh J (2021) \#allesdichtmachen. https://www.youtube.com/watch?v=HR4D_xJW8PQ. Zugegriffen: 15.12 .2021

Dor J (1999) The clinical Lacan. Other Press, New York

Dubois N (2021) \#allesdichtmachen. https://www.youtube.com/watch? $\mathrm{v}=$ OzlLDuf0bj8\&list= PLmb04gmYSul51BPqW1J93RgI1LYTCV6DF\&index=12. Zugegriffen: 15.12.2021

Duken K (2021) \#allesdichtmachen. https://www.youtube.com/watch? $\mathrm{v}=\mathrm{dCawGuje} 0 \mathrm{x} 0 \&$ list= PLmb04gmYSul51BPqW1J93RgI1LYTCV6DF\&index=8\&t=1s. Zugegriffen: 15.12 .2021

Düringer R (2021) \#allesdichtmachen. https://www.youtube.com/watch?v=DPymnYBdEeM. Zugegriffen: 15.12.2021 
Fink B (1995) The Lacanian subject: between language and Jouissance. Princeton University Press, Princeton

Fink B (1999) Clinical introduction to Lacanian psychoanalysis. Harvard University Press, Cambridge

Homer S (2016) Jacques Lacan. In: Elliott A, Prager J (Hrsg) The Routledge handbook of psychoanalysis in the social sciences and humanities. Routledge, New York, S 97-114

Jacobsen JT (2020) From neurotic citizen to hysteric security expert. Crit Stud Secur 8:46-58. https://doi. org/10.1080/21624887.2020.1735830

Johanssen J (2020) Social media and Coronavirus: paranoid-schizoid technology and pandemic? Hum Arenas. https://doi.org/10.1007/s42087-020-00162-2

Klein M (2019) Das Seelenleben des Kleinkindes und andere Beiträge zur Psychoanalyse, 11. Aufl. KlettCotta, Stuttgart

Klepec P (2016) On the mastery in the four „discourses“. In: Tomšič S, Zevnik A (Hrsg) Jacques Lacan: between psychoanalysis and politics. Routledge, New York, S 115-130

Küchenhoff J (2020) Die Coronakrise als Krise symbolischer Ordnungen und die Aufgaben der Psychoanalyse, das Sprechen zu wahren. https://ipa.world/IPA/en/IPA1/Webinars/Ein_neues_Unbehagen_ in_der_Kultur.aspx. Zugegriffen: 15.12.2021

Lacan J (1993) The psychoses 1955-1956. The seminar of Jacques Lacan, Bd. Book 3. W.W. Norton, New York

Lacan J (2007) The other side of psychoanalysis. The seminar of Jacques Lacan, Bd. Book XVII. W.W. Norton, New York

Merten T (2021) \#allesdichtmachen. https://www.youtube.com/watch? $\mathrm{v}=\mathrm{fHZCkyVlEmo \& list=}$ PLmb04gmYSu151BPqW1J93RgI1LYTCV6DF\&index=17. Zugegriffen: 15.12.2021

Müller R (2021) \#allesdichtmachen. https://www.youtube.com/watch?v=baYwUWYyo48. Zugegriffen: 15.12.2021

Nachtwey O, Schäfer R, Frei N (2020) Politische Soziologie der Corona-Proteste. https://osf.io/preprints/ socarxiv/zyp3f/. Zugegriffen: 15.12.2021

Osterode K (2021) \#allesdichtmachen. https://www.youtube.com/watch?v=u6QgZafp8G0\&list= PLmb04gmYSu151BPqW1J93RgI1LYTCV6DF\&index=25. Zugegriffen: 15.12.2021

Tukur U (2021) \#allesdichtmachen. https://www.youtube.com/watch?v=KQX79GqSAME\&t=1s. Zugegriffen: 15.12 .2021

Van Haute P, Geyskens T (2016) „Freud's dream“? Lacan and Oedipus. In: Tomšič S, Zevnik A (Hrsg) Jacques Lacan: between psychoanalysis and politics. Routledge, New York, S 47-61

Vanheule S (2016) Capitalist discourse, subjectivity and Lacanian psychoanalysis. Front Psychol 7:1-14. https://doi.org/10.3389/fpsyg.2016.01948

Wunder BK (2021) \#allesdichtmachen. https://allesdichtmachen.de/. Zugegriffen: 15.12.2021

Dr. phil. Nils F. Töpfer wissenschaftlicher Mitarbeiter an der Abteilung für Klinisch-Psychologische Intervention der Friedrich-Schiller-Universität Jena. Kandidat im DPG-IPV-Ausbildungsgang am Psychoanalytischen Institut Berlin e.V. Veröffentlichungen unter anderem zur psychoanalytischen Ausbildung und Supervision. Zuletzt (2021): „Where does it come from?“: A call for explicating implicit theories of interpretive and supervisory techniques in psychoanalytic supervision. Int Forum Psychoanal 30:46-55. 